\title{
Regular exercise during pregnancy did not affect physical growth or mental development of infants
}

Clapp JF 3rd, Simonian S, Lopez B, et al. The one-year morphometric and neurodevelopmental outcome of the offspring of women who continued to exercise regularly throughout pregnancy. Am J Obstet Gynecol 1998 Mar;178:594-9.

\section{Question}

What is the effect of regular, sustained, antigravitational exercise during pregnancy on the postnatal growth and neurodevelopment of infants?

\section{Design \\ Cohort study with follow up at 1 year.}

\section{Setting}

USA.

\section{Participants}

104 infants of women recruited from an ongoing study of exercise during pregnancy who had uncomplicated pregnancies, returned for follow up, and had a satisfactory evaluation at 1 year. All mothers were white, 25-38 years old, had family incomes in the upper $50 \%$, had a median of 17 years of education, and most $(91 \%)$ worked outside of the home. They all had well balanced diets, had 12-28\% body fat, and had no chronic illness or tobacco or drug use. Median parity was 2. Exclusion criteria were abnormal antenatal course (premature labour, pregnancy induced hypertension, abruptio placentae, or intrauterine growth retardation); intrapartum complications (eg, sepsis, fetal distress, or Apgar score $<7$ ); and diseases in infancy that could directly affect development (recurrent otitis media with effusion, feeding problems, surgery, or severe or protracted illness).

\section{Assessment of risk factors}

52 mothers were classified as exercisers (ran, did aeorobics, or used stair climbing machines $\geqslant 3$ times/wk for $>20 \mathrm{~min}$ at an intensity $>55 \%$ of their maximal performance during preg- nancy) and 52 were classified as physically active (intermittent activity such as golf, tennis, or hiking; infrequent activity; or no recreational exercise).

\section{Main outcome measures}

Within 24 hours of birth, a trained examiner measured infant weight; length; head, chest, and abdomen circumference; triceps, and subscapular skinfold thickness; and fat mass. Within 1 month of the child's first birthday, an examiner who was unaware of the mother's exercise status administered the original Bayley Scales of Infant Development (psychomotor and mental scales), and a different examiner repeated the morphometric measurements.

\section{Main results}

At birth, the infants of mothers who exercised had lower birth weights and less body fat (both $p=0.05$ ) than infants of mothers who were physically active, but did not differ for length, head circumference, or lean body mass. At 1 year, the groups did not differ for any of the morphometric assessments or for performance on the Bayley mental scale. Infants of women who exercised performed slightly better on the Bayley psychomotor scale $(\mathrm{p}=0.05)$.

\section{Conclusion}

Regular, sustained, antigravitational exercise during pregnancy did not affect infant physical growth or mental development at 1 year.

Sources of funding:National Institute of Child Health and Human Development; The Alexander von Humboldt Foundation; MetroHealth Medical Center.

For correspondence:Dr JF Clapp 3rd,Department of Obstetrics and Gynecology, MetroHealth Medical Center, 2500 MetroHealth Drive, Cleveland, OH 44109, USA. Fax +1 2127788847.

\section{Commentary}

Research suggests that regular aerobic exercise during pregnancy improves physical fitness, although a systematic review of the available trials found insufficient data to identify important risks or benefits to the mother or infant. ${ }^{1}$

This prospective study by Clapp et al followed up a small cohort of 104 physically fit women who became pregnant. The women were divided into 2 groups: those who continued to exercise regularly during pregnancy and those who were physically active but exercised intermittently. It might have been useful to include a group of women who did not exercise.

The avoidance of confounding variables assists the clarity of the study, but makes the findings less generalisable to other groups of pregnant women. Al- though the findings showed a statistically significant difference in body fat at birth and psychometric scores of infants at 1 year, these findings are not clinically significant. No consideration was given to the parity of the women, which may have had an effect on the birth weight of infants. The authors rightly point out the limitations of a small, non-representative cohort and are aware of the limitations of the Bayley Scales of Infant Development. All intelligence tests have different strengths and limitations and should be considered carefully before use.

Women who exercise regularly before becoming pregnant may seek advice from nurses and midwives on whether to continue exercising during pregnancy. This study will allow clinicians to reassure active women who fit the sample description that they can safely continue to be moderately active during pregnancy without harm to the fetus. Large randomised controlled trials are needed to adequately assess the potential effects of exercise during pregnancy for women and their babies.

Grace Edwards, RGN, RM, ADM, M Ed Regional Perinatal Surveys Manager Unit of Perinatal and Paediatric Epidemiology, University of Liverpool Liverpool, $U K$

1 Kramer MS. Regular aerobic exercise during pregnancy (Cochrane Review, latest version 29 Apr 1996). In: Cochrane Library. Oxford: Update Software. 